\title{
Driven maturation of embryonic stem cell-derived cardiomyocytes confers post-transplantation safety
}

Ji-Dong $\mathrm{Fu}^{1,2, *}$, Hung-fat Tse ${ }^{3, *}$, Chung-Wah Siu ${ }^{1,2,3, *}$, Jennifer C Moore, ${ }^{1,2}$, Deborah K Lieu, ${ }^{1,2}$, Song-Yan Liao ${ }^{3}$, Wing-Hon Lai ${ }^{3}$, Valeriy Timofeyez ${ }^{4}$, Ning $\mathrm{Li}^{4}$, Nipavan Chiamvimonvat ${ }^{4}$, Ronald A Li ${ }^{1,2,3,4,5}$

${ }^{I}$ Stem Cell Program and ${ }^{2}$ Department of Cell Biology and Human Anatomy, University of California, Davis, CA; ${ }^{3}$ Division of Cardiology, Department of Medicine, University of Hong Kong, China; ${ }^{4}$ Department of Internal Medicine, University of California, Davis, CA; Institute of Pediatric Regenerative Medicine, Shriners Hospital for Children of North America, Sacramento, CA, USA

While self-renewable, pluripotent human (h) embryonic stem cells (ESCs) can provide an unlimited source of donor cardiomyocytes (CMs) for myocardial repair, we showed in a preclinical large (porcine) animal transplantation model that their cardiac derivatives with immature properties are prime substrates for lethal cardiac arrhythmias. To address this, we developed an approach for driven maturation of hESC-derived CMs (hESCCMs) that renders the cellular electrophysiological phenotype adult-like and thus completely ablates post-transplantation ventricular tachycardias (VT)/fibrillation (VF).

Keywords: human embryonic stem cells, cardiomyocytes, maturation, electrophysiology, arrhythmogenicity

Cell Research (2008) 18:s132. doi: 10.1038/cr.2008.222; published online 4 August 2008

*These three authors contributed equally to this work.

Correspondence: Ronald Li

E-mail: ronaldli@ucdavis.edu 